\title{
NORMAL TERMS OF HAMILTONIAN SYSTEMS
}

\author{
P. WĄż \\ Institute of Astronomy, Nicolaus Copernicus University \\ Chopina 12/18, 87-100 Torúń, Poland
}

(Received June 22, 1995, revised version November 2, 1995)

\begin{abstract}
All the transformation matrices for a Hamiltonian with two and three degrees of freedom are found. They are calculated using algorithms for the linear normalization of Hamiltonian systems near the equilibrium point.
\end{abstract}

PACS numbers: $03.20 .+\mathrm{i}, 46.10 .+\mathrm{z}, 46.90 .+\mathrm{s}, 95.10 . \mathrm{Ce}$

\section{Introduction}

There are many possibilities of describing physical systems. Here we use Hamilton's formalism. This formalism is used in all the problems of celestial mechanics, in which the potential forces fulfill some general conditions. Here are some examples of such problems:

- The motion near any libration point in any restricted prablem;

- The rotational motion of artificial and natural satellites with the condition that they are rigid bodies;

- The motion of the planets and asteroids.

Let us consider a Hamiltonian system, which has $n$ degrees of freedom. Let

$$
\begin{aligned}
& q=\left(q_{1}, \ldots, q_{n}\right), \quad \boldsymbol{p}=\left(p_{1}, \ldots, p_{n}\right), \quad \boldsymbol{x}^{T}=(\boldsymbol{q}, \boldsymbol{p}), \\
& H=H(\boldsymbol{x})
\end{aligned}
$$

denote canonical variables, and a time independent Hamiltonian. Let us introduce a matrix $J$, which consists of $2 n$ rows and $2 n$ columns.

$$
J=-J^{T}=\left[\begin{array}{rr}
O_{n} & E_{n} \\
-E_{n} & 0_{n}
\end{array}\right],
$$

where $\boldsymbol{O}_{n}$ and $\boldsymbol{E}_{n}$ are null and unit matrices, respectively. Hamilton's equations of motion may be written in the following way:

$$
\frac{\mathrm{d} x}{\mathrm{~d} t}=J \frac{\partial H}{\partial \boldsymbol{x}} .
$$


We assume that this system has an equilibrium position $x^{r}$, i.e.

$$
\frac{\partial H}{\partial \boldsymbol{x}}\left(\boldsymbol{x}^{r}\right)=\boldsymbol{0} \text {. }
$$

One can assume that $x^{r}=0$. This can be achieved by using a canonical transformation - the linear translation

$$
x=x^{*}+x^{r}
$$

to move this point to zero. If one assumes the analyticity of the Hamiltonian function at this point one can get, by the expanding the Hamiltonian into MacLaurin's series about the canonical coordinates,

$$
H=H_{2}+H_{3}+\cdots+H_{m}+\cdots,
$$

where $H_{m}$ is a homogeneous polynomial of order $m$ in the canonical variables.

Equations (1) are, apart from a few particular cases, nonintegrable. Moreover there is no information about qualitative and quantitative solutions of this system. A very useful way to overcome these difficulties is a treatment called the normalization procedure. It is based on simplification of the terms in the Hamiltonian (2), using canonical transformations. After some steps of this procedure one can:

- establish the stability of the equilibrium position,

- find the approximate, analytical solution of equation (1),

- find the periodic and quasiperiodic families of the solutions,

- establish the initial conditions of the asymptotical solutions, which tend to the equilibrium position.

The aim of this paper is to formulate a general algorithm for the first step of the normalization procedure (called the linear normalization), which includes all possible Hamiltonian functions in the case of two and three degrees of freedom.

\section{Normal forms}

The whole normalization procedure depends very strongly on the $\mathrm{H}_{2}$ term, so it is very important to simplify it as much as possible. It is the first step of this procedure. In order to do that let us take the Hamiltonian system with the function $H=H_{2}$ [1]. The $H_{2}$ term is of the second order in $x$, so one can write it in the following way:

$$
H_{2}=\frac{1}{2} x^{T} h x,
$$

where

$$
h=\frac{\partial^{2} H}{\partial x^{2}}
$$

is a symmetric $2 n \times 2 n$ matrix. Hamilton's equations of motion for this system are linear

$$
\dot{x}=J h x .
$$

Let us now find the linear transformation

$$
x=N y,
$$


which is the canonical one

$$
N^{T} J N=J
$$

and transforms (3) into the normal form

$$
H_{2}^{*}=\frac{1}{2} y^{T} h^{*} y .
$$

Using the equations given above one gets the condition for the $N$ matrix

$$
N J h^{*}=J h N .
$$

This means that the matrices $\boldsymbol{J} h$ and $J h^{*}$ are similar to each other. Let us notice that the normal form (7) and $N$ depend on the structure of the matrix $J h$. The greater the simplification of $J h$ the more simple will be the resulted form of $H_{2}$. The most effective way to do that is to find the transformation, which transforms this matrix into Jordan's form (more general theory about Jordan's forms [2]). Generally this transformation does not have to be the canonical one, so we look for the matrix $N$ in the following form:

$$
\boldsymbol{N}=\boldsymbol{A B},
$$

where the matrix $\boldsymbol{A}$ transforms the matrix $\boldsymbol{J} h$ into Jordan's form, and $\boldsymbol{B}$ completes $\boldsymbol{A}$ in order to fulfill the condition (6). Let us consider now the practical using of the process given, ahead of looking for the normal forms. We look for the matrices, which transform $\boldsymbol{J} h$ into their simplest form. It is convenient to divide our problem into two parts. In the first part we consider all the Hamiltonian functions $H_{2}$, for which $\boldsymbol{J} h$ can be diagonalized, and in the second part the other ones. This division is very important, because the process of looking for the normal forms in these two cases is different.

\subsection{Case of simple elementary divisors}

In this part we consider all those Hamiltonians, for which the matrix $\boldsymbol{J h}$ can be transformed into a diagonal form.

\subsubsection{Way of constructing the matrix}

If $e_{k}$ and $e_{k+n}$ are eigenvectors of the matrix $J h$ :

$$
\begin{aligned}
& \boldsymbol{J} h \boldsymbol{e}_{k}=\lambda_{k} \boldsymbol{e}_{k}, \\
& \boldsymbol{J} \boldsymbol{h} \boldsymbol{e}_{k+n}=-\lambda_{k} \boldsymbol{e}_{k+n}, \quad k=1, \ldots, n,
\end{aligned}
$$

then the matrix $\boldsymbol{A}$ is constructed in the following way:

$$
A=\left[e_{1}, \ldots, e_{n}, e_{n+1}, \ldots, e_{2 n}\right] \text {. }
$$

\subsubsection{Structure of the matrix $\boldsymbol{A}^{T} \boldsymbol{J} \boldsymbol{A}$}

Let us put the term $\boldsymbol{N}=\boldsymbol{A} \boldsymbol{B}$ into the condition (6)

$$
B^{T} A^{T} J A B=\dot{J} .
$$

Let us consider the structure of the matrix $\boldsymbol{A}^{T} J \boldsymbol{A}$, which is interesting because of the way of construction of the matrix $\boldsymbol{A}$. Let us denote

$$
F=A^{T} J A \text {. }
$$


$\boldsymbol{J}$ is a skew-symmetric matrix and due to this fact the matrix $\boldsymbol{F}$ is also skew-symmetric. Transforming both sides of the following equation:

$$
e_{k}^{T} J^{2} h e_{l}=e_{k}^{T} h J^{2} e_{l}
$$

which is true for any $e_{j}$ (because $J^{2}=-E_{2 n}$ and the matrix $h$ is symmetric), one gets

$$
e_{k}^{T} J^{2} h e_{l}=J^{T} h^{T} e_{k} J e_{l}, \quad e_{k}^{T} J J h e_{l}=-J h e_{k} J e_{l} .
$$

$e_{k}$ are eigenvectors of the matrix $J h$, so one gets

$$
\boldsymbol{e}_{k}^{T} \boldsymbol{J} \lambda_{l} \boldsymbol{e}_{l}=-\lambda_{k} \boldsymbol{e}_{k}^{T} \boldsymbol{J} \boldsymbol{e}_{l}, \quad \lambda_{l} \boldsymbol{e}_{k}^{T} \boldsymbol{J} \boldsymbol{e}_{l}=-\lambda_{k} \boldsymbol{e}_{k}^{T} \boldsymbol{J} \boldsymbol{e}_{l} .
$$

These equations may be written in the following way:

$$
\left(\lambda_{k}+\lambda_{l}\right) f_{k l}=0, \quad f_{k l}=e_{k}^{T} J e_{l} .
$$

From this equation $f_{k l}=0$ in the case when

$$
\lambda_{k} \neq-\lambda_{l} \text {. }
$$

Knowing the structure of $\boldsymbol{A}$ and the conclusion given above one can write

$$
\boldsymbol{F}=\left[\begin{array}{rr}
\boldsymbol{O}_{n} & \boldsymbol{F}_{n}^{*} \\
-\boldsymbol{F}_{n}^{*} & \boldsymbol{O}_{n}
\end{array}\right],
$$

where $F_{n}^{*}$ is a diagonal matrix, with elements $f_{k k}^{*}=e_{k}^{T} J e_{k+n}$. None of these elements is equal to zero, because

$$
\operatorname{det} \boldsymbol{F}=\operatorname{det} \boldsymbol{A}^{T} \operatorname{det} \boldsymbol{J} \operatorname{det} \boldsymbol{A}=(\operatorname{det} \boldsymbol{A})^{2} \neq 0,
$$

since the matrix $\boldsymbol{A}$ consists of $2 n$ eigenvectors (linearly independent).

\subsubsection{Normalization matrix}

The construction of the matrix $B$ is the next step in the above algorithm. This matrix, multiplied by $\boldsymbol{A}$, gives the symplectic transformation. Let us assume that $\boldsymbol{B}=\boldsymbol{E}_{2 n}$. We have assumed nothing about the length of the vectors $\boldsymbol{e}_{k}$ and $e_{k+n}$, so it will be true that $\alpha_{k} \boldsymbol{e}_{k}$ and $\alpha_{k+n} e_{k+n}$ are also eigenvectors of the matrix $\boldsymbol{J} \boldsymbol{h}$. Let us write $\boldsymbol{A}$ in the following way:

$$
\boldsymbol{A}=\left[\alpha_{1} e_{1}, \ldots, \alpha_{k} e_{k}, \ldots, \alpha_{k+n} e_{k+n}, \ldots, \alpha_{2 n} e_{2 n}\right]
$$

and let $\alpha_{k}=\alpha_{k+n}$. Then the condition (6) transforms into the following equation:

$$
\alpha_{k}^{2} e_{k}^{T} J e_{k+n}=1
$$

where $k=1, \ldots, n$ (the left-hand side of this equation is not equal to zero, as shown earlier). Solving Eq. (12) one can find the $\alpha_{k}$ values. Then multiplying the $k$-th and the $(k+n)$-th vector by proper $\alpha_{k}$ values one gets the matrix $N$, which defines (by the relation $N^{T} h N=h^{*}$ following from Eqs. (6), (8)) the normal form of the Hamiltonian

$$
H_{2}^{*}=\sum_{j=1}^{n} \lambda_{j} y_{j} y_{j+n} .
$$

It is easy to notice this is the complex normal form, because generally $\lambda_{j}$ is complex (and also $x_{j}$ is complex for $j=1, \ldots, 2 n$ ). This form is much simpler and convenient for the next step of the normalization procedure than the real one. However, the Hamiltonian is real and after normalization we would like to get the 
real normal form. Such a form may be obtained from Eq. (13) by a standard, linear canonical transformation $Q^{-1}$ (Bruno [3]). This transformation is the reason for another condition, which the matrix $N$ has to fulfill. This condition will be fulfilled if $e_{k}^{T} J e_{k+n}>0$ for real $\lambda_{k}$ or $\operatorname{Im}\left(\boldsymbol{e}_{k}^{T} \boldsymbol{J} \boldsymbol{e}_{k+n}\right)<0$ (Im means here the imaginary part of the product) for imaginary $\lambda_{k}$. One has to exchange $\lambda_{k}$ with $\lambda_{k+n}$ and the $k$-th and $(k+n)$-th column of the matrix $\boldsymbol{A}$, when any of the two products has the opposite sign. By doing that the normal form (13) is the complex notation of the real normal form $[1,4]$.

\subsection{Multidimensional Jordan's boxes}

Let us consider now the second part of the problem. We look for the matrix $N$ in the following form: $\boldsymbol{N}=\boldsymbol{A} \boldsymbol{B}$ (as before). Also as before the matrix $\boldsymbol{A}$ transforms $J \boldsymbol{h}$ into Jordan's form and $\boldsymbol{B}$ completes $\boldsymbol{A}$, in this way the transformation defining by $N$ is the canonical one.

Let us introduce some notation. Let the symbol $e$ denote the columns of the matrix $\boldsymbol{A}$ and the eigenvectors or adjoint vectors of the matrix $\boldsymbol{J} h$. The subscript of $\boldsymbol{e}$ indicates the position of the column of the matrix $\boldsymbol{A}$. The superscript defines the connection of the adjoint vectors to the eigenvector with the same subscript. It is obvious that the eigenvectors of the $\boldsymbol{J} h$ matrix have only one symbol - the subscript. Let us denote $e_{l}$ as a vector connected to the eigenvalue $\lambda_{l}$, and $e_{l+n}$ to the eigenvalue $\lambda_{l}$. In the case of the elementary divisor $\lambda^{2\left(l+s_{l}\right)}$ (there are $1+2 s_{l}$ of adjoint vectors) we rewrite the last group of equations in another form. Let us assume that $\boldsymbol{e}_{l+n}^{l}$ is the $\left(s_{l}+1\right)$-th adjoint vector, $\boldsymbol{e}_{l+n+1}^{l}$ is the $\left(s_{l}+2\right)$-th one and so on. Thus

$$
\begin{aligned}
& J h e_{l+n}=e_{l+s_{l}}^{l}, \\
& J h e_{l+n+1}^{l}=e_{l+s_{l+1}}^{l}, \\
& \ldots \ldots \ldots \ldots \\
& J h e_{l+n+s_{l}}^{l}=e_{l+2 s_{l}}^{l} .
\end{aligned}
$$

Finally $\boldsymbol{A}$ may be written as follows:

$$
\boldsymbol{A}=\left[e_{1}, \ldots, e_{l}, e_{l+1}^{l}, \ldots, e_{l+s_{l}}^{l}, \ldots, e_{l+n}, e_{l+n+1}^{l+n}, \ldots, e_{l+n+s_{l}}^{l+n}, \ldots\right] .
$$

Let $\boldsymbol{B}=\boldsymbol{C}^{-1}$, where $\boldsymbol{C}$ transforms the matrix $\boldsymbol{J h ^ { * }}$ into Jordan's form. Thus we can write

$$
\boldsymbol{B} \boldsymbol{J} \boldsymbol{h}^{*}=\boldsymbol{G B}
$$

( $G$ is Jordan's form of the matrix $J h^{*}$ and $J h$ ). It is easy to find the general form of the matrix $B$, because one knows all Jordan's forms and the normal forms of $\boldsymbol{J} h$. Then one can find the elements of the matrix $\boldsymbol{B}$ by using the condition (6) and the fact that the matrix $N$ has to be real. Let us consider now the problem for three cases, when all the eigenvalues of $\boldsymbol{J} \boldsymbol{h}$

1. are equal to zero,

2. are real and different than zero,

3. are imaginary. 
These three cases describe all the Hamiltonian functions, which are contained in this section.

\subsubsection{Eigenvalues equal to zero}

Let us consider now only such Hamiltonian functions, for which the matrix $J h$ has only vanishing eigenvalues. This problem has been solved by Sokolski [5]. Based upon that paper one has:

Two degrees of freedom

There are the following normal forms, if all the $\boldsymbol{J} h$ values are equal to zero. These forms depend on the order of the matrix $h$ :

1. $H_{2}^{*}=0$ if $\operatorname{dim}(h)=0$,

2. $H_{2}^{*}=\frac{1}{2} \delta p_{1}^{* 2} \quad \delta= \pm 1 \quad$ if $\operatorname{dim}(h)=1$,

3. $H_{2}^{*}=\frac{1}{2} \delta_{1} p_{1}^{* 2}+\frac{1}{2} \delta_{2} p_{2}^{* 2} \quad \delta_{1}=\delta_{2}= \pm 1$ if $\operatorname{dim}(h)=3$,

4. $H_{2}^{*}=\frac{1}{2} \delta p_{1}^{* 2}+q_{1}^{*} p_{2}^{*} \quad \delta= \pm 1$ if $\operatorname{dim}(h)=4$.

The difference between Sokolski and our fourth relation follows from the fact that the basic material on which this paper is based on is Briuno's paper. In that paper for the pair of elementary divisors $\lambda^{l}$, where $l$ is an odd number, $H_{2}^{*}$ is given in the following form:

$$
H_{2}^{*}=\varepsilon \sum_{j=1}^{l-1} y_{j} y_{j+n+1} .
$$

For the elementary divisor $\lambda^{2 t}$

$$
H_{2}^{*}=\varepsilon \sum_{j=1}^{l-1} y_{j} y_{j+n+1}+\frac{1}{2} \delta y_{1}^{2} .
$$

One can assume that $\varepsilon=1$, because $\varepsilon$ is any complex number.

At the beginning let us consider the third and the fourth case.

Case 3

$$
\text { If }
$$

$$
J h e_{1}=0, \quad J h e_{2}=e_{1}, \quad J h e_{3}=0, \quad J h e_{4}=e_{3},
$$

then

$$
\boldsymbol{F}=\left[\begin{array}{clll}
0 & f_{12} & 0 & 0 \\
-f_{12} & 0 & 0 & 0 \\
0 & 0 & 0 & f_{34} \\
0 & 0 & -f_{34} & 0
\end{array}\right]
$$

where $f_{12} \neq 0, f_{34} \neq 0$, because $f_{12}^{2} f_{34}^{2}=\operatorname{det}(\boldsymbol{F})=\operatorname{det}(\boldsymbol{A})^{2} \neq 0$, and

$$
\boldsymbol{B}=\left[\begin{array}{cccc}
b_{1} & 0 & b_{3} & 0 \\
0 & 0 & \delta_{1} b_{1} & 0 \\
0 & b_{2} & 0 & b_{4} \\
0 & 0 & 0 & \delta_{2} b_{2}
\end{array}\right]
$$

From the condition (6) one gets

$$
\delta_{1} b_{1}^{2} \dot{f}_{12}=1, \quad \delta_{2} b_{2}^{2} f_{34}=1 .
$$


Assuming, for simplicity, $b_{3}=b_{4}=0$ one gets

where

$$
N\left[b_{1} e_{1}, \quad b_{2} e_{3}, \quad \delta_{1} b_{1} e_{2}, \delta_{2} b_{2} e 4\right]
$$

Case 4

$$
b_{1}=\left(e_{1}^{T} J e_{2}\right)^{-1 / 2}, \quad b_{2}=\left(e_{3}^{T} J e_{4}\right)^{-1 / 2}, \quad \delta_{1}=\operatorname{sgn}\left(e_{1}^{T} J e_{2}\right), \quad \delta_{2}=\operatorname{sgn}\left(e_{3}^{T} J e_{4}\right)
$$

Let

$$
J h e_{1}=0, \quad J h e_{2}=e_{1}, \quad J h e_{3}=e_{2}, \quad J h e_{4}=e_{3},
$$

then

$$
\boldsymbol{F}=\left[\begin{array}{clcl}
0 & 0 & 0 & f_{14} \\
0 & 0 & -f_{14} & 0 \\
0 & f_{14} & 0 & f_{34} \\
-f_{14} & 0 & -f_{34} & 0
\end{array}\right]
$$

$\boldsymbol{F}$ is a non-singular matrix so $f_{14} \neq 0$. The $\boldsymbol{B}$ matrix in this case can be written in the following form:

$$
\boldsymbol{B}=\left[\begin{array}{cccc}
b_{1} & b_{2} & b_{3} & b_{4} \\
b_{2} & 0 & \delta b_{1} & -b_{3} \\
0 & 0 & \delta b_{2} & -\delta b_{1} \\
0 & 0 & 0 & -\delta b_{2}
\end{array}\right],
$$

and the condition (6) is fulfilled if

$$
\begin{aligned}
& -\delta b_{2} b_{2} f_{14}=1, \\
& b_{1} b_{1} f_{14}-2 \delta b_{2} b_{3} f_{14}-b_{2} b_{2} f_{34}=0 .
\end{aligned}
$$

Assuming that $b_{1}=0$, we have

and

$$
N\left[b_{2} e_{2}, \quad b_{2} e_{1}, \quad \delta b_{2} e_{3}+b_{3} e_{1}, \quad-\delta b_{2} e_{4}-b_{3} e_{2}\right]
$$

$$
b_{4}=0, \quad \delta=-\operatorname{sgn}\left(e_{1}^{T} J e_{4}\right), \quad b_{2}=\left(e_{1} J e_{4}\right)^{-1 / 2}, \quad b_{3}=\frac{b_{2} e_{3}^{T} J e_{4}}{-2 \delta e_{1}^{T} J e_{4}} .
$$

The first case is omitted, because it was described in the preceding section. It is useful to notice that if $\operatorname{dim}(h)=4$ then $\mathrm{H}_{2}$ is already written in the simplest form. So the process of simplification is not needed in this case. The second case is omitted, because it is a combination of the first and the third one.

\section{Three degrees of freedom}

There are eight different normal forms for this number of degrees of freedom.

1. $H_{2}^{*}=0$ if $\operatorname{dim}(h)=0$,

2. $H_{2}^{*}=\frac{1}{2} \delta p_{1}^{* 2}$ if $\operatorname{dim}(h)=1$, where $\delta= \pm 1$;

3. $H_{2}^{*}=\frac{1}{2} \delta_{1} p_{1}^{* 2}+\frac{1}{2} \delta_{2} p_{2}^{* 2}$ if $\operatorname{dim}(h)=2$, where $\delta_{1}=\delta_{2}= \pm 1$,

4. $H_{2}^{*}=\frac{1}{2} \delta p_{1}^{* 2}+q_{1}^{*} p_{2}^{*}$ if $\operatorname{dim}(h)=3$, where $\delta= \pm 1$, or

5. $H_{2}^{*}=\frac{1}{2} \delta_{1} p_{1}^{* 2}+\frac{1}{2} \delta_{2} p_{2}^{* 2}+\frac{1}{2} \delta_{3} p_{3}^{* 2}$, where $\delta_{1}=\delta_{2}=\delta_{3}= \pm 1$, 
6. $H_{2}^{*}=q_{1}^{*} p_{2}^{*}+q_{2}^{*} p_{3}^{*} \quad$ if $\operatorname{dim}(h)=4$, or

7. $H_{2}^{*}=\frac{1}{2} \delta_{1} p_{1}^{* 2}+q_{2} p_{3}+\frac{1}{2} \delta_{2} p_{2}^{* 2}$, where $\delta_{1}=\delta_{2}= \pm 1$,

8. $H_{2}^{*}=q_{1}^{*} p_{2}^{*}+q_{2}^{*} p_{3}^{*}+\frac{1}{2} \delta p_{2}^{* 2}$ if $\operatorname{dim}(h)=5$, where $\delta= \pm 1$.

At first let us try to find the normalization matrices only for the 6-th and 8-th cases.

Case 6

For this case we have the following equations:

$J h e_{1}=0, \quad J h e_{2}=e_{1}, \quad J h e_{3}=e_{2}, \quad J h e_{4}=0, \quad J h e_{5}=e_{4}, \quad J h e_{6}=e_{5}$,

so

$$
\boldsymbol{F}=\left[\begin{array}{ccclcl}
0 & 0 & 0 & 0 & 0 & f_{16} \\
0 & 0 & f_{23} & 0 & -f_{16} & f_{26} \\
0 & -f_{23} & 0 & f_{16} & -f_{26} & f_{36} \\
0 & 0 & -f_{16} & 0 & 0 & 0 \\
0 & f_{16} & f_{26} & 0 & 0 & f_{56} \\
-f_{16} & -f_{26} & -f_{36} & 0 & -f_{56} & 0
\end{array}\right]
$$

and $f_{16} \neq 0$. Looking for the matrix $\boldsymbol{B}$, according to the algorithm one gets

$$
\boldsymbol{B}=\left[\begin{array}{llllcl}
b_{1} & b_{2} & b_{3} & b_{4} & b_{5} & b_{6} \\
b_{2} & b_{3} & 0 & 0 & -b_{4} & -b_{5} \\
b_{3} & 0 & 0 & 0 & 0 & b_{4} \\
b_{10} & b_{8} & b_{9} & b_{7} & b_{11} & b_{12} \\
b_{8} & b_{9} & 0 & 0 & -b_{7} & -b_{11} \\
b_{9} & 0 & 0 & 0 & 0 & b_{7}
\end{array}\right] .
$$

The normalization conditions can be written as

$$
\begin{aligned}
& b_{7} b_{3} f_{16}-b_{4} b_{9} f_{16}=1, \\
& -2 b_{2} b_{9} f_{16}-2 b_{3} b_{9} f_{26}+2 b_{3} b_{8} f_{16}-b_{3}^{2} f_{23}-b_{9} f_{56}=0, \\
& b_{2} b_{7} f_{16}+b_{4} b_{3} f_{23}+b_{11} b_{3} f_{16}+b_{7} b_{3} f_{26}+b_{4} b_{8} f_{16}-b_{5} b_{9} f_{16}-b_{4} b_{9} f_{26} \\
& +b_{7} b_{9} f_{56}=0, \\
& 2 b_{5} b_{7} f_{16}-2 b_{5} b_{9} f_{26}-2 b_{4} b_{11} f_{16}-b_{4}^{2} f_{23}-b_{7}^{2} f_{56}=0, \\
& b_{7} b_{1} f_{16}+b_{7} b_{2} f_{26}+b_{2} b_{11} f_{16}+b_{4} b_{2} f_{23}+b_{3} b_{5} f_{23}+b_{12} b_{3} f_{16}+b_{11} b_{3} f_{26} \\
& +b_{7} b_{3} f_{36}-b_{4} b_{10} f_{16}-b_{8} b_{5} f_{16}+b_{4} b_{8} f_{26}+b_{8} b_{7} f_{56}-b_{6} b_{9} f_{16}+b_{9} b_{4} f_{26} \\
& -b_{4} b_{9} f_{36}+b_{11} b_{9} f_{56}=0 .
\end{aligned}
$$

Then by assuming (for algebraic simplicity) $b_{6}=b_{4}=b_{9}=b_{12}=0$, $b_{10}=b_{11}=0$ one gets

$$
b_{3}=1, \quad b_{7}=\frac{1}{f_{16}}, \quad b_{2}=\frac{-b_{3} f_{26}}{f_{16}}, \quad b_{8}=\frac{b_{3} f_{23}}{2 f_{16}}, \quad b_{5}=\frac{b_{7} f_{56}}{2 f_{16}},
$$


Case 8

$$
b_{10}=\frac{b_{8} b_{5} f_{16}-b_{8} b_{7} f_{56}-b_{2} b_{7} f_{26}-b_{3} b_{5} f_{23}-b_{7} b_{3} f_{36}}{b_{7} f_{16}},
$$

In this case

$$
J h e_{1}=0, \quad J h e_{2}=e_{1}, \quad J h e_{3}=e_{2}, \quad J h e_{4}=e_{3}, \quad J h e_{5}=e_{4}, \quad J h e_{6}=e_{5},
$$

so

$$
\boldsymbol{F}=\left[\begin{array}{clclcl}
0 & 0 & 0 & 0 & 0 & f_{16} \\
0 & 0 & 0 & 0 & -f_{16} & 0 \\
0 & 0 & 0 & f_{16} & 0 & f_{36} \\
0 & 0 & -f_{16} & 0 & -f_{36} & 0 \\
0 & f_{16} & 0 & f_{36} & 0 & f_{56} \\
-f_{16} & 0 & -f_{36} & 0 & -f_{56} & 0
\end{array}\right] .
$$

The element $f_{16} \neq 0$, because the matrix $\boldsymbol{F}$ is non-singular. The matrix $\boldsymbol{B}$ for this form is the following:

$$
\boldsymbol{B}=\left[\begin{array}{cccccc}
b_{1} & b_{2} & b_{3} & b_{4} & b_{5} & b_{6} \\
b_{2} & b_{3} & 0 & \delta b_{1} & -b_{4} & -b_{5} \\
b_{3} & 0 & 0 & \delta b_{2} & -\delta b_{1} & b_{4} \\
0 & 0 & 0 & \delta b_{3} & -\delta b_{2} & \delta b_{1} \\
0 & 0 & 0 & 0 & -\delta b_{3} & \delta b_{2} \\
0 & 0 & 0 & 0 & 0 & \delta b_{3}
\end{array}\right] .
$$

The condition (6) is fulfilled when

$$
\begin{aligned}
& \delta b_{3} b_{3} f_{16}=1 \\
& 2 \delta b_{3} b_{1} f_{16}+\delta b_{3} b_{3} f_{36}-\delta b_{2} b_{2} f_{16}=0, \\
& b_{2} b_{2} f_{36}+2 \delta b_{2} b_{4} f_{16}+2 \delta b_{3} b_{5} f_{16}-b_{3} b_{3} f_{56}-2 b_{1} b_{3} f_{36}-b_{1} b_{1} f_{16}=0 .
\end{aligned}
$$

Assuming $b_{2}=0$ and $b_{4}=0$ one gets

$$
N^{T}=\left[\begin{array}{c}
b_{1} e_{1}+b_{3} e_{3} \\
b_{3} e_{2} \\
b_{3} e_{2} \\
\delta b_{1} e_{2}+\delta b_{3} e_{4} \\
b_{5} e_{1}-\delta b_{1} e_{3}-\delta b_{3} e_{5} \\
-b_{5} e_{2}+\delta b_{1} e_{4}+\delta b_{3} e_{6}
\end{array}\right]
$$


where

$$
\begin{aligned}
& \delta=\operatorname{sign}\left(f_{16}\right), \quad b_{3}=\left(f_{16}\right)^{-1 / 2}, \quad b_{1}=\frac{-f_{36} b_{3}}{2 f_{16}}, \\
& b_{5}=\frac{b_{1} b_{1} f_{16}+2 b_{3} b_{1} f_{36}+b_{3} b_{3} f_{56}}{2 \delta f_{16} b_{3}} .
\end{aligned}
$$

We do not consider other cases, because they may be reduced to combinations of the previous cases.

\subsubsection{Real eigenvalues}

For the pair of elementary divisors $\left(\lambda^{*}-\lambda\right)^{l}$ and $\left(\lambda^{*}+\lambda\right)^{l}$, where $\lambda$ is the real eigenvalue, the normal form given by Briuno [2] may be written in the following way:

$$
H_{2}^{*}=\lambda \sum_{j=1}^{l} y_{j} y_{j+n}+\varepsilon \sum_{j=1}^{l-1} y_{j} y_{j+n+1} .
$$

Let us consider only two cases. In the first one

$$
J h e_{1}=\lambda_{1} e_{1}, \quad J h e_{2}=\lambda_{1} e_{2}+e_{1}, \quad J h e_{3}=-\lambda_{1} e_{3}, \quad J h e_{4}=-\lambda_{1} e_{4}+e_{3},
$$

and so

$$
\begin{aligned}
& \boldsymbol{F}=\left[\begin{array}{clcl}
0 & 0 & 0 & f_{14} \\
0 & 0 & -f_{14} & f_{24} \\
0 & f_{14} & 0 & 0 \\
-f_{14} & -f_{24} & 0 & 0
\end{array}\right], \\
& \boldsymbol{B}=\left[\begin{array}{llll}
b_{1} & b_{2} & 0 & 0 \\
b_{2} & 0 & 0 & 0 \\
0 & 0 & b_{3} & b_{4} \\
0 & 0 & 0 & -b_{3}
\end{array}\right]
\end{aligned}
$$

From the condition (6) it follows that

$$
\begin{aligned}
& -f_{14} b_{3} b_{2}=1, \\
& -b_{1} b_{3} f_{14}-b_{2} b_{4} f_{14}-f_{24} b_{2} b_{3}=0 .
\end{aligned}
$$

Now let $b_{1}=0$, then

$$
b_{2}=\left(f_{14}\right)^{-1 / 2}, \quad \delta=\operatorname{sgn}\left(f_{14}\right), \quad b_{3}=-\delta\left(f_{14}\right)^{-1 / 2}, \quad b_{4}=\frac{f_{24} b_{3}}{-f_{14}} .
$$

The matrix $N$ may be written as

$$
N=\left[\begin{array}{llll}
b_{2} e_{2}, & b_{2} e_{1}, & b_{3} e_{3}, & b_{4} e_{3}-b_{3} e_{4}
\end{array}\right] .
$$

In the second case

$$
\begin{aligned}
& J h e_{1}=\lambda_{1} e_{1}, \quad J h e_{2}=\lambda_{1} e_{2}+e_{1}, \quad J h e_{3}=\lambda_{1} e_{3}+e_{2}, \\
& J h e_{4}=-\lambda_{1} e_{4}, \quad J h e_{5}=-\lambda_{1} e_{5}+e_{4}, \quad J h e_{6}=-\lambda_{1} e_{6}+e_{5},
\end{aligned}
$$


so

$$
\boldsymbol{F}=\left[\begin{array}{clclcl}
0 & 0 & 0 & 0 & 0 & f_{16} \\
0 & 0 & 0 & 0 & -f_{16} & f_{26} \\
0 & 0 & 0 & f_{16} & -f_{26} & f_{36} \\
0 & 0 & -f_{16} & 0 & 0 & 0 \\
0 & f_{16} & f_{26} & 0 & 0 & 0 \\
-f_{16} & -f_{26} & -f_{36} & 0 & 0 & 0
\end{array}\right]
$$

and $f_{16} \neq 0$. The matrix $B$ may be written in the following way:

$$
\boldsymbol{B}=\left[\begin{array}{cccccc}
b_{1} & b_{2} & b_{3} & 0 & 0 & 0 \\
b_{2} & b_{3} & 0 & 0 & 0 & 0 \\
b_{3} & 0 & 0 & 0 & 0 & 0 \\
0 & 0 & 0 & b_{4} & b_{5} & b_{6} \\
0 & 0 & 0 & 0 & -b_{4} & -b_{5} \\
0 & 0 & 0 & 0 & 0 & b_{4}
\end{array}\right]
$$

The transformation matrix fulfills the condition (6) if

$$
\begin{aligned}
& b_{3} b_{4} f_{16}=1, \\
& b_{4} b_{2} f_{16}+b_{3} b_{5} f_{16}+b_{3} b_{4} f_{26}=0 \\
& b_{4} b_{1} f_{16}+b_{2} b_{5} f_{16}+b_{4} b_{2} f_{26}+b_{6} b_{3} f_{16}+b_{5} b_{3} f_{26}+b_{4} b_{3} f_{36}=0 .
\end{aligned}
$$

One can assume for algebraic simplicity $b_{1}=b_{5}=0$, then

$$
b_{3}=\frac{1}{f_{16}}, \quad b_{4}=1, \quad b_{2}=\frac{-b_{3} f_{26}}{f_{16}}, \quad b_{6}=\frac{b_{4}\left(f_{26}^{2} / f_{16}-f_{36}\right)}{f_{16}}
$$

and

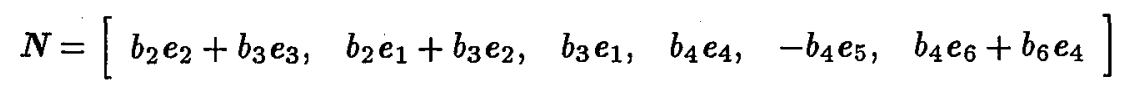

\subsubsection{Imaginary eigenvalues}

In this section we consider the pair of elementary divisors $\left(\lambda^{*}-\lambda\right)^{l}$ and $\left(\lambda^{*}+\lambda\right)^{l}$, where $\lambda$ is the imaginary eigenvalue $(\lambda=\mathrm{i} \beta)$. The normal form for this case, given by Briuno [2], is the following:

$$
\begin{aligned}
H_{2}^{*} & =\frac{1}{2} \beta \sum_{j=1}^{l}\left(y_{j} y_{l+1-j}+y_{j+n} y_{l+n+1-j}\right) \\
& +\frac{1}{2} \delta \sum_{j=1}^{l-1}\left(y_{j} y_{l-j}+y_{1+j+n} y_{l+n+1-j}\right),
\end{aligned}
$$

where $\delta= \pm 1$. Let us consider (like for the real eigenvalues) two cases: when $l$ is equal to two and three. The matrices $\boldsymbol{F}$ are the same like in the case of real eigenvalues. It is worth noticing that for $l=2$ the element $f_{14}$ is the real value, and $f_{24}$ the imaginary one, but if $l=3$ both $f_{16}$ and $f_{36}$ are imaginary and $f_{26}$ 
is real. Let the power of elementary divisor be equal to two, and let the matrix $\boldsymbol{A}$ be constructed according to the recipe described at the beginning of this section. Then

$$
\boldsymbol{B}=\left[\begin{array}{cccc}
b_{1} & b_{2} & -\mathrm{i} \delta b_{2} & -\mathrm{i} \delta b_{1} \\
\mathrm{i} b_{2} & 0 & 0 & \delta b_{2} \\
b_{9} & b_{10} & \mathrm{i} \delta b_{10} & \mathrm{i} \delta b_{9} \\
-\mathrm{i} b_{10} & 0 & 0 & \delta b_{10}
\end{array}\right]
$$

The normalization conditions have the form

$$
\begin{aligned}
& 2 \delta b_{2} b_{10} f_{14}=1, \\
& 2 \delta b_{1} b_{10} f_{14}+2 \delta b_{2} b_{9} f_{14}+\mathrm{i} 2 \delta b_{10} b_{2} f_{24}=0 .
\end{aligned}
$$

So the matrix $\boldsymbol{N}$ is given by

$$
N^{T}=\left[\begin{array}{c}
b_{1} e_{1}+\mathrm{i} b_{2} e_{2}+b_{9} e_{3}-\mathrm{i} b_{10} e_{4} \\
b_{2} e_{1}+b_{10} e_{3} \\
-\mathrm{i} \delta b_{2} e_{2}+\mathrm{i} \delta e_{3} \\
-\mathrm{i} \delta b_{1} e_{1}+\delta b_{2} e_{2}+\mathrm{i} \delta b_{9} e_{3}+\delta b_{10} e_{4}
\end{array}\right]
$$

where

and

$$
\delta=\operatorname{sgn}\left(f_{14}\right), \quad b_{2}=b_{10}=\left(2 \delta f_{14}\right)^{-1 / 2}, \quad b_{1}=b_{9}=-\frac{\mathrm{i} b_{2} f_{24}}{2 f_{14}}
$$

$$
e_{3}=\overline{e_{1}}, \quad e_{4}=\overline{e_{2}} .
$$

The straight line over $e_{k}$ means the coupling of the vector. For $l=3$

$$
\boldsymbol{B}=\left[\begin{array}{cccccc}
b_{1} & b_{2} & b_{3} & -\mathrm{i} \delta b_{3} & -\mathrm{i} \delta b_{2} & -\mathrm{i} \delta b_{1} \\
\mathrm{i} b_{2} & \mathrm{i} b_{3} & 0 & 0 & \delta b_{3} & \delta b_{2} \\
-b_{3} & 0 & 0 & 0 & 0 & \mathrm{i} \delta b_{3} \\
b_{19} & b_{20} & b_{21} & \mathrm{i} \delta b_{21} & \mathrm{i} \delta b_{20} & \mathrm{i} \delta b_{19} \\
-\mathrm{i} b_{20} & -\mathrm{i} b_{21} & 0 & 0 & \delta b_{21} & \delta b_{20} \\
-b_{21} & 0 & 0 & 0 & 0 & -\mathrm{i} \delta b_{21}
\end{array}\right]
$$

The condition (6) is fulfilled if

$$
\begin{aligned}
& -2 \mathrm{i} \delta b_{21} b_{3} f_{16}=1, \\
& -2 \mathrm{i} \delta b_{21} b_{2} f_{16}-2 \mathrm{i} \delta b_{20} b_{3} f_{16}+2 \delta b_{3} b_{21} f_{26}=0, \\
& -2 \mathrm{i} \delta b_{21} b_{1} f_{16}-2 \mathrm{i} \delta b_{20} b_{2} f_{16}-2 \mathrm{i} \delta b_{19} b_{3} f_{16} \\
& \quad+2 \delta b_{2} b_{21} f_{26}+2 \delta b_{20} b_{3} f_{26}+2 \mathrm{i} \delta b_{3} b_{21} f_{36}=0 .
\end{aligned}
$$

Finally

$$
N^{T}=\left[\begin{array}{c}
b_{1} e_{1}-b_{3} e_{3}+\mathrm{i} b_{2} e_{2}+b_{19} e_{4}-\mathrm{i} b_{20} e_{5}-b_{21} e_{6} \\
b_{2} e_{1}+\mathrm{i} b_{3} e_{2}+b_{20} e_{4}-\mathrm{i} b_{21} e_{5} \\
b_{3} e_{1}+b_{21} e_{4} \\
-\mathrm{i} \delta b_{3} e_{1}+\mathrm{i} \delta b_{21} e_{4} \\
-\mathrm{i} \delta b_{2} e_{1}+\delta b_{3} e_{2}+\mathrm{i} \delta b_{20} e_{4}+\delta b_{21} e_{5} \\
-\mathrm{i} \delta b_{1} e_{1}+\mathrm{i} \delta b_{3} e_{3}+\delta b_{2} e_{2}+\mathrm{i} \delta b_{19} e_{4}+\delta b_{20} e_{5}-\mathrm{i} \delta b_{21} e_{6}
\end{array}\right]
$$


and

$$
\begin{aligned}
& \delta=\operatorname{sgn}\left(\operatorname{Im} f_{16}\right), \quad b_{3}=b_{21}=\left(2 \operatorname{Im}\left(f_{16}\right)\right)^{-1 / 2} \\
& b_{2}=b_{20}=\frac{b_{3} f_{26}}{2 \mathrm{i} f_{16}}, \quad b_{1}=b_{19}=\frac{\mathrm{i} b_{3} f_{36}+1.5 b_{2} f_{26}}{2 \mathrm{i} f_{16}}, \\
& e_{4}=\overline{e_{1}}, \quad e_{5}=\overline{e_{2}}, \quad e_{6}=\overline{e_{3}} .
\end{aligned}
$$

Like for the real eigenvalues these two cases are sufficient to get the complete solution of the Hamiltonian function, for which the $\boldsymbol{J} h$ matrices have degenerate eigenvalues.

\section{Conclusion}

None of the above ways of finding $N$ matrices depend upon the number of degrees of freedom. One can easily find, using the algorithms described in this paper, the transformation matrices for any Hamiltonian (fulfilling the initial conditions) with any degrees of freedom. It is enough to construct the $\boldsymbol{B}$ and $\boldsymbol{F}$ matrices for such systems of the elementary divisor (according to the properties of the $\boldsymbol{J} \boldsymbol{h}$ matrices), which we have not considered in the case of two and three degrees of freedom.

\section{Acknowledgments}

The author is indebted to Andrzej J. Maciejewski for several discussions. He also thanks prof. Brian Wybourne and Dorota Bielińska-Wąz for their linguistic help. This work was supported by the grant No. 221049203 of the Committee for Scientific Research.

\section{References}

[1] A.J. Maciejewski, K. Goździewski, Astrophys. Space Sci. 179, 1 (1991).

[2] F.R. Gantmacher, Theory of Matrices, Nauka, Moskwa 1988.

[3] A.D. Bruno, Usp. Mat. Nauk 43, 22 (1988) (in Russian).

[4] A.P. Markeev, Libration Points in Celestial Mechanics and Astrodynamics, Nauka, Moskwa 1978 (in Russian).

[5] A.G. Sokolsky, Prikl. Mat. Mekh. 45, (3) (1981). 